SCIENTIFIC LETTER

\title{
Is the value of QT dispersion a valid method to foresee the risk of sudden death? A study in Becker patients
}

\author{
Ge Nigro, G Nigro, L Politano, L Santangelo, V R Petretta, L Passamano, F Panico, \\ F De Luca, A Montefusco, L I Comi
}

Heart 2002;87: 156-157

S udden cardiac death is a dramatic, undesirable event that can often result from cardiomyopathies. To investigate the validity of the QTdispersion (QTd) in revealing regional heterogeneity of repolarisation, with consequent possibility of sudden death, ${ }^{1}$ we evaluated ECGs of patients affected by Becker muscular dystrophy (BMD). This is an X linked recessive muscular dystrophy caused by dystrophin anomalies in striated muscles with myocardial involvement ${ }^{2-4}$ and consequent dilated cardiomyopathy, ventricular arrhythmias and, in $30 \%$ of cases, sudden cardiac death. ${ }^{56}$

\section{METHODS}

The study was retrospective using the clinically validated database of ECGs and echocardiograms (echo recorded together with the ECG) from 30 BMD patients (mean (SD) age 25 (10) years) with variable stages of myocardial involvement, and 26 healthy, age matched controls. All subjects underwent a physical examination, blood analyses, and $\mathrm{M}$ mode and two dimensional echocardiography. The diagnosis of the type of muscular dystrophy in Becker patients was confirmed by DNA analysis (polymerase chain reaction) and by reduced dystrophin labelling from the immunohistochemical examination of biopsy samples.

We excluded the ECGs from subjects with ST-T anomalies on the 12 lead ECG, and with sustained ventricular arrhythmias at 24 hour Holter monitoring, electrolyte imbalance, or QRS duration longer than $120 \mathrm{~ms}$ and from subjects under cardiovascular treatment.

The standard 12 lead ECG, recorded at a paper speed of $25 \mathrm{~mm} / \mathrm{s}$ (gain $10 \mathrm{~mm}=1 \mathrm{mV}$ ) on a three channel recorder, was analysed for each subject by a blinded observer using a digitiser connected to a computerised system. QRS, RR, QT, JT (the latter obtained from the formula QT-QRS) were measured over three consecutive cycles. When U waves were present, the end of the $\mathrm{T}$ wave was considered the nadir of the curve between the $\mathrm{T}$ and the $\mathrm{U}$ waves. The QTd was defined as the difference between the maximum and minimum QT values. QT and JT were further corrected according to Bazett's formula. Extra and post-extra systolic cycles were excluded from the analysis.

For each parameter, a mean value was calculated for each lead dividing their sums by the total number of leads-at least

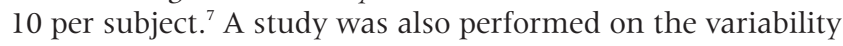
of QT measurements: 20 ECGs were coded, duplicated, and measured twice by the same blind observer. Percentage difference in QT measurements for the same ECG ranged from 1.5$2.8 \%$ for intra-observer variability. In addition, ejection fraction and fibre shortening were calculated from echocardiograms performed at the same time with ECG recordings.

Among the Becker patients, eight died from sudden cardiac death within 12 weeks after the registration of the examined ECG. There were no differences in ventricular arrhythmias, or echocardiographic findings between survivors and patients who died suddenly.
Table 1 Mean (SD) values and significance of the electrocardiographic and echocardiographic parameters in 30 Becker patients and in 26 age and sex matched controls

\begin{tabular}{llll}
\hline & Becker patients & Control group & $\mathrm{p}$ Value \\
\hline Age & $25(10$ & $30(7)$ & $\mathrm{NS}$ \\
Sex & Men & Men & \\
Ejection fraction & $53.00(9.98)$ & $61.80(3.08)$ & 0.0001 \\
Fibre shortening & $27.23(5.92)$ & $32.28(2.09)$ & 0.0001 \\
RR interval & $799.10(121.86)$ & $864.24(97.78)$ & $\mathrm{NS}$ \\
QRS interval & $108.81(15.98)$ & $96.10(9.64)$ & 0.003 \\
JT interval & $277.3(31.77)$ & $271.48(21.10)$ & $\mathrm{NS}$ \\
JTc value & $299.29(49.68)$ & $290.81(13.41)$ & $\mathrm{NS}$ \\
QT interval & $386.14(35.42)$ & $367.43(20.46)$ & $\mathrm{NS}$ \\
QTc value & $436(32.30)$ & $393(13.48)$ & 0.0001 \\
QTd interval & $110.62(28.76)$ & $59.14(19.07)$ & 0.0001 \\
\hline
\end{tabular}

The relationship between echocardiographic parameters and QTd in Becker patients were statistically evaluated using the linear regression and correlation analysis. Electrocardiographic data are presented as mean (SD). Mann-Whitney non-parametric test and Student $t$ test for unpaired data were chosen to compare the electrocardiographic data from the two groups. The same statistical tests were used to compare the ECG and echo data obtained from the eight BMD patients who died suddenly and those from the remaining BMD population. A probability value of $\mathrm{p}<0.05$ for both Mann-Whitney nonparametric test and Student $t$ test for unpaired data was considered significant. No significant linear correlation was found between echocardiographic parameters (ejection fraction, fibre shortening) and QTd in Becker patients.

A scattergram was made to compare the values of QTd calculated in Becker patients who died suddenly, the survivors and control group.

\section{RESULTS}

Findings from the electrocardiographic and echocardiographic parameters in BMD patients and in controls are presented in table 1. No significant difference between the two groups was found in RR, QT, JT, and JTc. A significant increase $(p<0.003)$ in $Q R S$ duration and a highly significant difference $(p=0.0001)$ in ventricular recovery time dispersion indexes (QTc, QTd) were observed in Becker patients compared to normal subjects.

The results obtained comparing the electrocardiographic and echocardiographic parameters from the eight Becker patients who suddenly died and from those who survived lend

Abbreviations: BMD, Becker muscular dystrophy; QTd, QTdispersion 


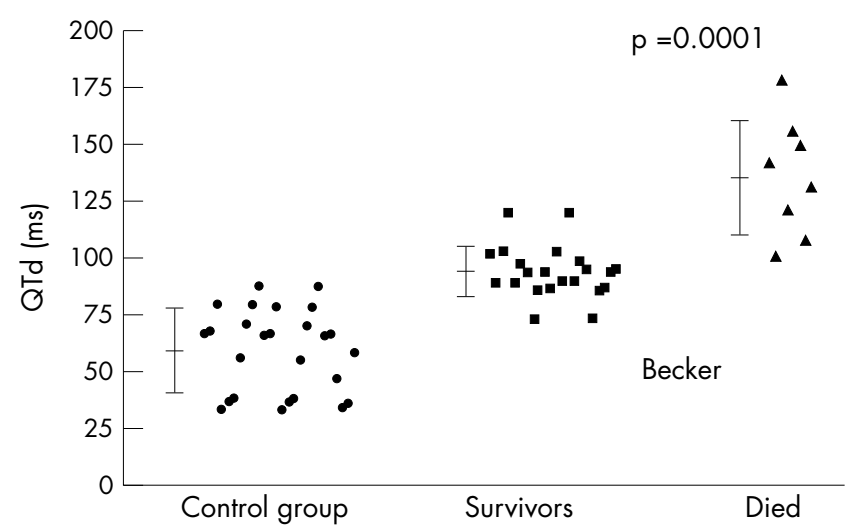

Figure 1 Comparison between QTd values in those Becker muscular dystrophy patients who died suddenly, those who survived, and controls. Horizontal bars indicate the mean values, and vertical bars the standard deviation.

weight to the study. In fact, although no sustained ventricular arrhythmias $(>30)$ were seen on the 24 hour Holter monitoring-recorded at the same time of the examined ECG-in patients who died and no differences were noted in their clinical conditions, or in echocardiographic parameters compared to the survivors (one patient with heart failure among the eight who died suddenly and one among the survivors $)$, a highly significant difference $(p=0.0001)$ is seen between the QTd in the two groups. Figure 1 shows that none of the Becker patients who died had QTd values $<100 \mathrm{~ms}$, and six of them had values higher than the maximum QTd value recorded in the survivors. This latter observation shows the need for careful monitoring of Becker patients; a 24 hour Holter must be performed every month when the QTd value reaches $100 \mathrm{~ms}$ and, if it reveals runs of ventricular arrhythmias, pharmacological treatment with antiarrhythmic drugs (amiodarone) should be given. If pharmacotherapy is ineffective, a cardioverter-defibrillator should be implanted.
Our findings demonstrate that the analysis of dispersion of ventricular activation in the 12 lead surface ECG (QTd) was significantly increased in BMD patients; this parameter is principally related to non-homogeneous electrical activity. The ease with which the QTd can be calculated, and the possibility of using the QTd value as a valid parameter to predict the risk of sudden death, may provide opportunities through antiarrhythmic drug administration, or an implanted cardioverter-defibrillator, to prevent this dramatic event.

\section{Authors' affiliations}

Ge Nigro, L Santangelo, F Panico, A Montefusco, Electrophysiologic Service of Cardiological Department, Second Naples University, Naples, Italy

G Nigro, L Politano, V R Petretta, L Passamano, F De Luca, L I Comi, Cardiomyologic and Genetic Section, Department of Internal and Experimental Medicine, Second Naples University, Naples, Italy

Correspondence to: Dr Lucia I Comi, Vaile dei Pini 101, 80131 Naples, Italy; lucia_ines.comi@unina2.it

\section{Accepted 8 October 2001}

\section{REFERENCES}

1 Fu GS, Meissner A, Simon R. Repolarization dispersion and sudden cardiac death in patients with impaired left ventricular function. Eur Heart J 1997:18:281-9.

2 de Visser M, de Vogt WG, la Riviere GU. The heart in Becker muscular dystrophy. Muscle Nerve 1992;15:591-5.

3 Nigro G, Comi LI, PolitanoL, et al Evaluation of the cardiomyopathy in Becker muscular dystrophy. Muscle Nerve 1995;18:283-91.

4 Maeda M, Nakao S, Miyzato H, et al. Cardiac dystrophin abnormalities in Becker muscular dystrophy assessed by endomyocardial biopsy. Am Heart J 1995; 129:702-7.

5 Zaidi $\mathbf{M}$, Robert A, Fesler R, et al. Dispersion of ventricular repolarization in dilated cardiomyopathy. Eur Heart J 1997;18:1129-34.

6 Grimm W, Steden U, Menz V, et al. QT dispersion and arrhythmic events in idiopathic dilated cardiomyopathy. Am J Cardiol 1996;78:458-61.

7 Malik $M$, Betchvarov VN. Measurement, interpretation and clinical potential of QT dispersion. J Am Coll Cardiol 2000;36:1749-66.

\section{IMAGES IN CARDIOLOGY}

\section{Magnetic resonance angiography in the evaluation of aortic pseudoaneurysm}

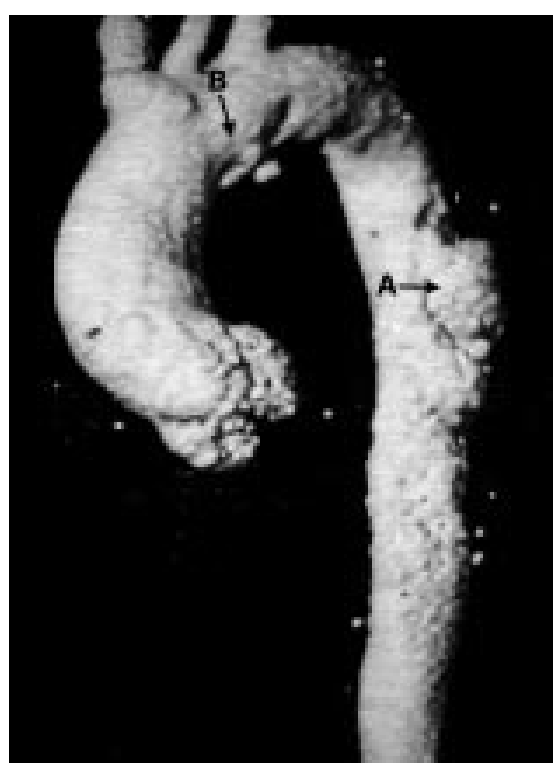

agnetic resonance angiography (MRA) and transoesophageal echocardiography together are very useful imaging techniques in the evaluation of thoracic aortic pseudoaneuIrysms. A 63 year old hypertensive man was admitted to a community hospital by his primary care physician with three weeks history of night sweats, chills, fever, fatigue, and generalised myalgia. Blood cultures done in the outpatient clinic revealed group D salmonella bacteraemia. Transthoracic echocardiogram, abdominal computed tomography (CT), and ultrasound were normal. CT of the chest showed increased density within the anterior mediastinum, suspicious of an aortic pseudoaneurysm.

MRA of the thoracic aorta (left) showed two infected pseudoaneurysms, one in the descending aorta starting below the aortic arch (A), extending $7 \mathrm{~cm}$ caudally, and another smaller one in the lesser curve of the aortic arch (B). The latter (B) was missed on transoesophageal echocardiography, although the former (A) was seen clearly. Preoperative coronary angiography showed critical three vessel disease. The patient successfully underwent resection of aneurysms with Dacron patch repair of the lesser curve and Hemashield tube graft repair of the descending aorta. He was given ceftriaxone for a week and later switched to levofloxacin, which he has been taking long term.

Once invariably a fatal condition, infected aortic pseudoaneurysms can now be cured in most cases if diagnosed early and treated with appropriate surgical technique and antibiotic. Both MRA and transoesophageal echocardiography complement each other in the evaluation of this condition. 